\title{
ẢNH HƯởNG CỦA NHIẸT Độ, Độ MặN TỚI SỰ THAY ĐỔI VậN TỐC ÂM TẠI KHU VỰC VỊNH BẮC Bộ
}

\author{
NGUYẼ̃N VĂN CƯƠNG ${ }^{(1)}$, NGUYẼ̃N GIA TRỌNG(2) \\ (1) Trung tâm Trắc địa và Bản đồ Biển \\ (2) Trường đại học Mỏ - Địa chất
}

\section{Tóm tắt:}

Vận tốc âm thanh trong nước biển phụ thuộc vào nhiệt độ $(T)$, độ mặn (S) và áp suất $P$ (hay độ sâu $D$ ) và thay đổi theo từng mùa trong năm đối với một vùng biển rộng. Nội dung bài báo trình bày kết quả khảo sát xác định vận tốc âm tại một số vị trí thuộc vùng biển Vịnh Bắc Bộ với dữ liệu đo nhiệt độ và độ mặn ở các độ sâu từ Om đến $70 \mathrm{~m}$ trong khoảng thời gian từ 01/01/2006 đến 01/12/2006. Kết quả khảo sát cho thấy mức độ biến thiên của vận tốc âm trong thời gian một năm tại khu vực Vịnh Bắc Bộ có thể lên tới $27.2 \mathrm{~m} / \mathrm{s}$. Đây là điều cần lưu ý trong công tác xác định vận tốc âm khi triển khai đo đạc bằng các thiết bị thủy âm ở Vịnh Bắc Bộ.

\section{Mở đầu}

Xác định chính xác vận tốc âm thanh trong môi trường nước sẽ làm tăng độ chính xác kết quả đo đạc bằng các thiết bị sử dụng nguyên lý thủy âm như máy đo sâu hồi âm, thiết bị định vị thủy âm, thiết bị dò thủy âm quét sườn .v.v... Vận tốc âm thanh trong các vùng biển và đại dương phụ thuộc vào nhiệt độ $(T)$, độ mặn $(S)$ và áp suất $(P)$ và do đó nó cũng thay đổi theo mùa trong năm và phụ thuộc vào vị trí của điểm xét trên vùng biển. Áp suất luôn liên quan tới độ sâu cho nên không thay đổi đáng kể theo từng mùa hoặc từ nơi này sang nơi khác; nó gần như chỉ thay đổi theo độ sâu lớp nước. Tuy nhiên, nhiệt độ và độ mặn (T / S) lại có sự biến đổi theo cả thời gian và không gian.

Mặt cắt vận tốc âm thanh SSP (Sound Speed Profile) được xây dựng dựa trên kết quả tính toán vận tốc âm theo các giá trị quan trắc nhiệt độ $(\mathrm{T})$ và độ mặn $(\mathrm{S})$ tại các lớp nước có độ sâu khác nhau. Để tính toán vận tốc âm theo nhiệt độ, độ mặn và độ sâu, chúng ta có thể sử dụng các công thức thực nghiệm do một số tác giả đưa ra như công thức của Wilson [7], công thức Del Grosso
[5], công thức Mackenzie [3], công thức Coppens [4], công thức do Tồ chức Giáo dục, Khoa học và Văn hoá Liên Hợp Quốc (UNESCO) đề xuất [6], công thức Medwin [9],... Các công thức trên đều có quy định khoảng giá trị của các tham số phù hợp cho việc sử dụng. Đối với vùng biển nước ta, độ sâu tối đa thường nhỏ hơn $1000 \mathrm{~m}$, nên thường sử dụng công thức Medwin để tính toán vận tốc âm. Công thức Medwin có dạng:

$$
\begin{aligned}
& v=1449.2+4.6 \mathrm{~T}-0.055 \mathrm{~T}^{2}+0.00029 \mathrm{~T}^{3} \\
& +(1.34-0.010 \mathrm{~T})(\mathrm{S}-35)+0.016 \mathrm{D}
\end{aligned}
$$

Công thức này áp dụng phù hợp với các khoảng giá trị tham số như sau:

- Nhiệt độ trong khoảng: $0 \leq \mathrm{T} \leq 35^{\circ} \mathrm{C}$;

- Độ mặn trong khoảng: $0 \leq S \leq 45$ ppt (phần nghìn);

- Độ sâu trong khoảng: $0 \leq \mathrm{D} \leq 1000 \mathrm{~m}$

Trong tài liệu [2], Xavier Lurton dựa trên công thức Medwin để thể hiện mối quan hệ giữa vận tốc âm với nhiệt độ và độ mặn tại lớp nước bề mặt $(z=0)$. Mối quan hệ đó được thể hiện trền biểu đồ hình 1.

\footnotetext{
Ngày nhận bài: 01/3/2018, ngày chuyển phản biện: 08/3/2018, ngày chấp nhận phản biện: 16/3/2018, ngày chấp nhận đăng: 20/3/2018
} 


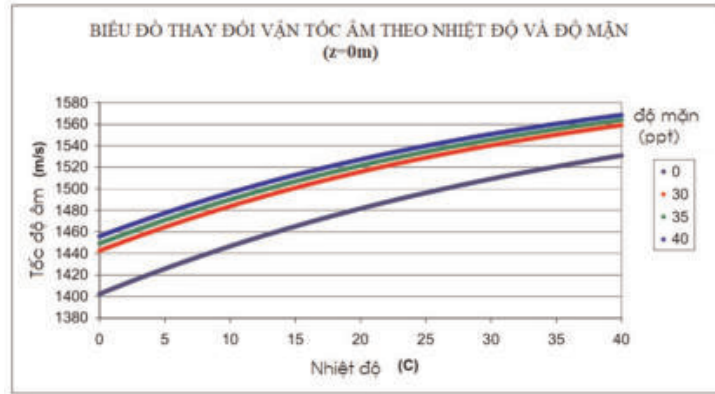

Hình 1: Biếu đồ thay đối vận tổc âm theo nhiệt độ và độ mặn tại z=0m[2]

Từ biểu đồ trên có thể nhận thấy ảnh hưởng của độ mặn nước biển tới vận tốc âm là không lớn nhưng ảnh hưởng của nhiệt độ tới vận tốc âm là đáng kể, đặc biệt ở giải nhiệt độ từ $0^{\circ} \mathrm{C}$ đến $30^{\circ} \mathrm{C}$.

Để xác định vận tốc âm trên khu vực biển Vịnh Bắc Bộ, chúng tôi sử dụng thiết bị CTD Guildline đo độ dẫn C (độ mặn), nhiệt độ $T$ và độ sâu $D$, thiết bị này cho phép đo độ mặn trong khoảng từ 0 ppt đến 40 ppt với độ chính xác \pm 0.3 ppt, đo nhiệt độ trong khoảng từ $-3^{\circ} \mathrm{C}$ đến $+39^{\circ} \mathrm{C}$ với độ chính xác $\pm 0.03^{\circ} \mathrm{C}$.

\section{Số liệu quan trắc nhiệt độ $(T)$ và độ mặn (S)}

Với mục tiêu xác định ảnh hưởng của nhiệt độ và độ mặn tới kết quả tính toán xác định vận tốc âm trong khu vực biển Vịnh Bắc Bộ, chúng tôi sử dụng dữ liệu đo (CTD) trong thời gian từ 1/1/2006 đến 1/12/2006 tại 3 vị trí đặc trưng của Vịnh Bắc Bộ là:

Vị trí $\mathrm{A}$ (phía Bắc Vịnh Bắc Bộ): Tọa độ: $B=20.5, L=108.0$ và độ sâu $40 \mathrm{~m}$.

Vị trí $\mathrm{B}$ (giữa Vịnh Bắc Bộ): Tọa độ: $B=19.5, L=107.0$ và độ sâu $50 \mathrm{~m}$.

Vị trí C (Nam Vịnh Bắc Bộ): Tọa độ: $B=18.0 L=107.25$ và độ sâu $70 \mathrm{~m}$.

Vị trí các điểm quan trắc $(A, B, C)$ được thể hiện trên bản đồ ảnh vệ tinh tại hình 2 .

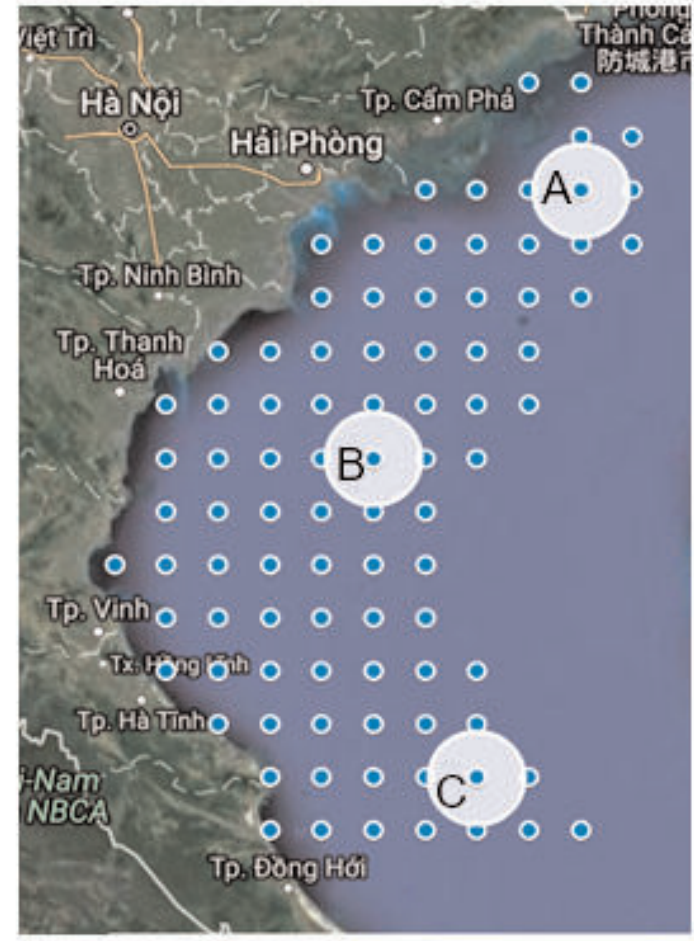

Hình 2: Vị trí các điểm quan trắc trên Vịnh Bắc Bộ

Số liệu đo nhiệt độ và độ mặn của 12 tháng trong năm 2006 tại các vị trí $A, B, C$ được thống kê trong các bảng sau: (Xem bảng 1, 2, 3)

Từ số liệu đo được thống kê trên các bảng trên có thể nhận thấy rằng sự biến đổi nhiệt độ $T$ theo các tháng trong năm trên bề mặt là khá lớn (khoảng $9.5^{\circ} \mathrm{C}$ ), biến đổi độ mặn theo các tháng trong năm là không nhiều (0.6ppt). Ở phía Bắc Vịnh Bắc Bộ, nhiệt độ có sự biến thiên nhiều hơn so với phía Nam Vịnh Bắc Bộ.

\section{Tính toán vận tốc âm theo số liệu quan trắc}

Dựa vào công thức Medwin, chúng tôi tính toán xác định vận tốc âm theo nhiệt độ, độ mặn và độ sâu cho 12 tháng trong năm 2006. Các giá trị vận tốc âm tại các lớp nước và vận tốc âm trung bình (Vtb) được thể hiện trên các bảng 4,5,6. 
Bảng 1: Số liệu đo nhiệt độ, độ mặn tại vị trí A (năm 2006)

\begin{tabular}{|c|c|c|c|c|c|c|c|c|c|c|c|c|c|c|c|c|c|c|c|c|c|c|c|c|}
\hline fháng & & & & & & & & & & & & & & & & & & & & & & & & \\
\hline 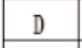 & $I$ & - & I & $s$ & I & $s$ & I & $s$ & I & s & I & $s$ & I & s & I & $s$ & I & $s$ & 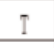 & $s$ & $\therefore$ & $s$ & ${ }_{0}$ & $s$ \\
\hline 0 & 20.0 & 32.5 & 18.8 & 32.5 & 21.2 & 33.3 & 22.8 & 33.5 & 5.5 & 33.6 & 29.3 & 31.4 & 9.5 & 31.3 & 29.5 & 31.2 & 27.6 & 32.2 & 27.1 & 32.2 & 26.0 & 32.2 & 22.8 & 32.3 \\
\hline & 0.0 & 2.6 & 1 & 32.5 & 9 & 33.3 & 4 & 33.5 & 1 & 33.6 & 2.2 & 31.5 & 9.4 & 31.5 & 9.4 & 31.3 & 27.6 & 32.2 & 27.1 & 32.2 & 26.0 & 32.2 & 22.8 & 32. \\
\hline & 9.9 & 6 & 7 & 32.5 & 6 & 33.3 & 0 & 33.4 & 8 & 33.6 & 1 & 31 & 3 & 31.6 & 3 & 31.5 & 17.6 & 32.2 & 27.1 & 32.3 & 6.0 & 2.2 & 22.8 & 32.3 \\
\hline & 9.9 & 6 & 6 & 2 & 3 & 33.3 & 1 & 33.4 & 4 & 33.6 & 1 & 31.8 & 9.3 & 31.8 & 9.3 & 31.7 & 27.6 & 32.5 & 27.1 & 32. & 26.0 & 22.2 & 22.7 & 32. \\
\hline 8 & 19.9 & 6 & 18.5 & 1.6 & 19.9 & 33.3 & 21.3 & 33.4 & 24.0 & 33.6 & 29.0 & 32.0 & 29.2 & 31.9 & 29.2 & 31.9 & 27.6 & 32.3 & 27.1 & 32.3 & 26.0 & 32.3 & 22.7 & 32.3 \\
\hline 10 & 19.9 & 6 & 18.4 & 1.6 & 6 & 33.3 & 9 & 33.4 & 1 & 33.5 & 28.9 & 32.2 & 29.1 & 32.1 & 29.1 & 32.1 & 27.5 & 32.3 & 27.1 & 32 & 26.0 & 5 & 2.7 & 32 \\
\hline 15 & 19.8 & 52.0 & 18.3 & 2600 & 19.4 & 33.3 & 20.6 & 33. & 2 & 33.5 & 8.5 & 32.6 & 8.7 & 32. & 8.9 & 32 & 27.5 & 32 & 27.0 & 32.4 & 26.0 & 32.3 & 22.7 & 2. \\
\hline 20 & 19.8 & 6 & 18.2 & 32.6 & 19.2 & 33.3 & 20.4 & 33.4 & 22.7 & 33.5 & 8.0 & 32. & 8.2 & 32. & 8.6 & 32. & 27.4 & 32. & 27.0 & 32.5 & 26.1 & 32.4 & 22.7 & 32. \\
\hline 25 & 19.8 & 32.7 & 18.1 & 32.6 & 19.1 & 33.3 & 20.2 & 33.4 & 22.3 & 33.4 & 17.5 & 33.0 & 7.8 & 33. & 8.4 & 33.0 & 27.3 & 32. & 27.0 & 32.5 & 26.0 & 32.5 & 22.7 & 32. \\
\hline 30 & 9.8 & 32.7 & 8.0 & 32.6 & 9 & 33.3 & 9 & 33.3 & 1.1 & 33. & 7.2 & 33. & 7.5 & 33. & 8.1 & 33. & 27.1 & 32 & 26.8 & 32.8 & 15.9 & 32.6 & 22.7 & 32. \\
\hline 35 & 19.8 & 52. & 18.0 & 32.7 & 18.9 & 33. & 19.9 & 3j. & 9 & jj. & 9 & 33. & 7.1 & 33. & 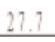 & 33 & 26.8 & 32.9 & 26.6 & 1.9 & 1 & 32.7 & 22.7 & 32. \\
\hline 40 & 19.8 & 32.7 & 18.0 & 32.7 & 18.8 & 33.3 & 19.7 & 33.3 & 21.7 & 33.4 & 6.5 & 33.3 & 6.7 & 33.2 & 3 & 33.2 & 26.5 & 33.1 & 26.3 & 33.1 & 25.4 & 9 & 2.7 & \\
\hline
\end{tabular}

Bảng 2: Số liệu đo nhiệt độ, độ mặn tại vị trí B (năm 2006)

\begin{tabular}{|c|c|c|c|c|c|c|c|c|c|c|c|c|c|c|c|c|c|c|c|c|c|c|c|c|c|c|}
\hline & & & & & & & & & & & & & & & & & & & & & & & 1 & 11 & & \\
\hline D & $T$ & pos & 1 & $\mathrm{~S}$ & & & & & & & 10 & & $T_{\sigma}$ & & $T_{c}$ & & & $\sigma_{0}$ & & $s_{0}$ & & & $T_{T}$ & $\mathrm{~s}$ & & \\
\hline & & 32.8 & & & & & & & & & & & & & .1 & & & & & & .1 & & 5.4 & & 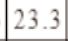 & \\
\hline & & & & & & & & & & & & & & & & & & & & & & & .4 & & & $1-$ \\
\hline & & & & & & & & & & & 33.6 & . & & & & & .0 & & 3.4 & & & & .4 & & & 3 \\
\hline & & & & & & & & & & & 33 & s. & 13 & & & & .0 & & .4 & & & & 4.4 & & $\begin{array}{l}3.3 \\
\end{array}$ & 2 . \\
\hline & & 3 & & & & & & & & 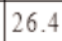 & .6 & 28. & $\begin{array}{ll}03 \\
\end{array}$ & & \begin{tabular}{l|l}
.8 & 3.
\end{tabular} & 2.9 & 8.9 & & 3 & & & 32. & .4 & & 3.3 & 2. \\
\hline II & 0.6 & 2.9 & 19.6 & 5 & & & & & 3.4 & . & 33.6 & 28. & 0. & 8 & \begin{tabular}{l|l|l}
.7 & 3
\end{tabular} & 3 & 8.8 & & 3.3 & & 0 & 32. & 5.4 & & 3. & 2 . \\
\hline 19 & .6 & 3 & 19.5 & 2.5 & s & & & & 3.4 & 8.6 & 33.6 & 27. & 1 & 27 & \begin{tabular}{|l|l|l}
.8 &
\end{tabular} & 3.2 & 28.2 & & 7.9 & 32.7 & ${ }^{9}$ & 32.8 & 4 & & & 2. \\
\hline 11 & 0.6 & 33.0 & 19.4 & 33.0 & 9.7 & 33. & 22 & & 3.4 & 24.9 & 33.6 & 26. & \begin{tabular}{l|l}
1 & 33 \\
\end{tabular} & 26 & \begin{tabular}{l|l|l}
.9 & 3 \\
\end{tabular} & 3.3 & 27.5 & 33.2 & 27.6 & 32.9 & 26.9 & 32.8 & 4.4 & t. & 23.3 & 3 \\
\hline 15 & 0.5 & 33.0 & 19.4 & 33.0 & 9.6 & \begin{tabular}{l|l}
633 \\
6
\end{tabular} & 21 & & 3.5 & 24.2 & 33.6 & \begin{tabular}{|l|l|}
65. \\
6
\end{tabular} & \begin{tabular}{l|l}
4 & 33
\end{tabular} & 26 & \begin{tabular}{l|l|l}
.23 \\
\end{tabular} & 3.5 & 26.9 & 33.4 & 27.2 & 33.0 & 6.7 & 32.9 & .4 & 4 & 23.3 & 33. \\
\hline & 0.5 & 33.0 & 19.3 & 33.0 & 9.5 & & 21 & \begin{tabular}{l|l|l}
4 & 3
\end{tabular} & 3.4 & 23.6 & 6 & \begin{tabular}{|l|l|}
64. \\
\end{tabular} & & 25 & 6 & 3.5 & 26.4 & & 6.8 & 33.2 & & 33.0 & 4 & & 3.2 & 33. \\
\hline it & 0.5 & 33.1 & 19.3 & & & & 21 & & 3.4 & 23.4 & 6 & 24. & & 25 & 4 & 3.5 & 26.0 & & 6.4 & 3 & & 33.1 & 3 & & 3.2 & 33. \\
\hline 4 & 0.5 & & 19.2 & & & & 21 & $\begin{array}{ll}0.03 \\
\end{array}$ & 3.5 & 23.2 & & \begin{tabular}{|l|l}
64. \\
\end{tabular} & & 25 & .2 & 3.5 & 25.7 & & 26.1 & & 0 & 33.2 & 2 & & 3.2 & 32. \\
\hline 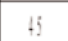 & 0.4 & 33.1 & 19.2 & & & & 20 & & & 23.0 & & \begin{tabular}{l|l}
64. \\
6
\end{tabular} & & 25 & & & 25.5 & & 25.6 & {$[33.4$} & 23.7 & 33.3 & 25.0 & J. & 23.2 & 2. \\
\hline & & & & & & & & & & & & & & & & & & & & & & & & & & \\
\hline
\end{tabular}

Giá trị vận tốc âm nhỏ nhất vào tháng 2 $\left(V_{\min }=1513.6 \mathrm{~m} / \mathrm{s}\right)$, lớn nhất vào tháng 7 , tháng $8\left(V_{\max }=1540,8 \mathrm{~m} / \mathrm{s}\right)$. Như vậy, sự biển đổi vận tốc âm trong năm tại vị trí $A$ (Bắc Vịnh Bắc Bộ) là $27,2 \mathrm{~m} / \mathrm{s}$. Nếu so sánh vận tốc âm trung bình thì giá trị trung bình nhỏ nhất vào tháng $2,\left(\mathrm{~V}_{\mathrm{tb}}=1514,4 \mathrm{~m} / \mathrm{s}\right)$ và tháng 8 có giá trị trung bình lớn nhất $\left(V_{\mathrm{tb}}=1540,2 \mathrm{~m} / \mathrm{s}\right)$, chênh lệch vận tốc trung bình giữa tháng 2 và tháng 8 là $25,8 \mathrm{~m} / \mathrm{s}$. (Xem hinh 3)

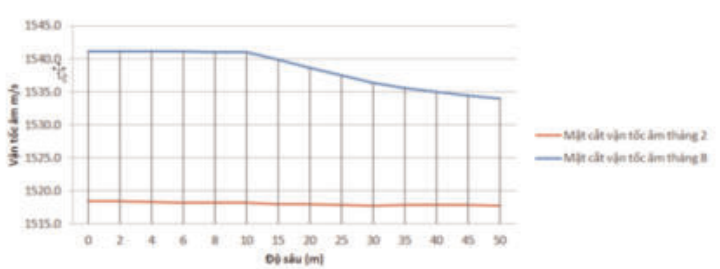

Hình 3: Mặt cắt vận tồc âm tháng 2 và tháng 8 (vị trí $A$ )

Theo mặt vận tốc âm trong tháng 2 và tháng 8 tại vị trí $\mathrm{A}$, biên độ thay đổi vận tốc 
Bảng 3: Số liệu đo nhiệt độ, độ mặn tại vị trí C (năm 2006)

\begin{tabular}{|c|c|c|c|c|c|c|c|c|c|c|c|c|c|c|c|c|c|c|c|c|c|c|c|c|}
\hline háng & & 1 & & 2 & & 3 & 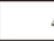 & 4 & 3 & & 6 & & 7 & & & 8 & 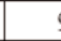 & 7 & 1 & 0 & 1 & 1 & 1. & \\
\hline & $\mathrm{T}$ & S & $\mathrm{T}$ & $\mathrm{S}$ & $\mathrm{T}$ & $\mathrm{S}$ & $\mathrm{T}$ & $\mathrm{S}$ & $\mathrm{T}$ & $S$ & $\mathrm{~T}$ & S & $\mathrm{T}$ & S & $\mathrm{T}$ & $\mathrm{S}$ & $\mathrm{T}$ & S & $\mathrm{T}$ & $\mathrm{S}$ & $\mathrm{T}$ & S & $\mathrm{T}$ & $\mathrm{c}$ \\
\hline 0 & 21.9 & 33.4 & 21.5 & 33.3 & 22.8 & 33.2 & 24.8 & 33.4 & 27.1 & 33.2 & 28.3 & 33.2 & 28.9 & 33.0 & 28.9 & 33.1 & 28.6 & \begin{tabular}{|l}
32.4 \\
\end{tabular} & \begin{tabular}{|l}
17.8 \\
\end{tabular} & $\begin{array}{l}33.5 \\
\end{array}$ & \begin{tabular}{|l}
26.5 \\
\end{tabular} & 32.3 & 24.5 & 33.2 \\
\hline 1 & 12.9 & 33.4 & .5 & 33.3 & 18 & 33.2 & 24.8 & 33.4 & 26.9 & 33.2 & 18.1 & 33.3 & 28.8 & 33.0 & \begin{tabular}{|l}
18.8 \\
\end{tabular} & 33.16 & 28.5 & 32.5 & \begin{tabular}{|l}
17.8 \\
\end{tabular} & \begin{tabular}{|l}
33.5 \\
\end{tabular} & 26.5 & 32.5 & 24.5 & 33.2 \\
\hline 4 & 21.9 & 33.4 & 21.4. & 33.3 & 12.7 & 33.2 & 24.7 & 33.4 & 26.7 & 33.3 & 27.9 & 33.4 & 28.6 & 33.1 & 28.7 & 33.1 & 18.4 & 32.6 & \begin{tabular}{|l}
17.8 \\
\end{tabular} & \begin{tabular}{|l}
33.5 \\
\end{tabular} & 26.5 & 32.7 & 24.5 & 33.2 \\
\hline 6 & 12.9 & 33.5 & 21.. & 33.3 & 21.9 & 33.2 & 24.6 & 33.4 & 26.6 & 33.2 & 21.9 & 33.4 & 28.4 & 33.2 & 28.5 & 33.2 & 28.4 & 32.7 & \begin{tabular}{|l}
17.9 \\
\end{tabular} & 33.4 & 26.5 & 32.9 & 24.5 & 33.2 \\
\hline 8 & 21.9 & 33.5 & 21.4 & 33.3 & 12.6 & 33.2 & 24.5 & 33.5 & 26.4 & 33.3 & 217.5 & 33.5 & 28.2 & 33.2 & 28.4 & 33.2 & 28.3 & 32.8 & 11.9 & 33.4 & \begin{tabular}{|l}
26.5 \\
\end{tabular} & 33.1 & 24.5 & 33.3 \\
\hline 11 & 12.9 & 3.5 & 21.4 & 33.3 & 22.6 & 33.3 & 24.4 & 33.5 & 26.2 & 33.3 & 27.3 & 33.5 & 28.10 & 33.3 & 28.2 & 33.3 & 28.2 & 32.9 & \begin{tabular}{|l}
17.6 \\
\end{tabular} & 33.4 & \begin{tabular}{|l}
26.5 \\
\end{tabular} & 33.3 & 24.5 & 33.3 \\
\hline 15 & 12.9 & 33.5 & 21.3 & 33.3 & 22.4 & 33.4 & 23.8 & 33.5 & 25.1 & 33.5 & 25.8 & 33.6 & 26.4 & 33.4 & 27.0 & 33.5 & 27.5 & 33.2 & 121.4 & 33.4 & \begin{tabular}{|l}
26.5 \\
\end{tabular} & 33.4 & 24.5 & 33.4 \\
\hline 11 & 21.9 & 33.5 & $21 .$. & 33.4 & $2 \ldots .2$ & 33.5 & 23.1 & 33.6 & 24.0 & 33.6 & 24.4 & 33.6 & 24.9 & 33.6 & 25.9 & 33.6 & 26.8 & 33.5 & \begin{tabular}{|l}
27.1 \\
\end{tabular} & 33.4 & \begin{tabular}{|l}
26.5 \\
\end{tabular} & 33.5 & 24.5 & 33.5 \\
\hline 15 & 21.9 & 33.6 & 21.1 & 33.5 & $\begin{array}{l}21.9 \\
\end{array}$ & 33.8 & 21.5 & 33.7 & 23.1 & 33.7 & 23.4 & 33.7 & 23.8 & 33.9 & 24.8 & 33.7 & 25.9 & 33.6 & \begin{tabular}{|l|l} 
\\
\end{tabular} & 33.5 & 26.4 & 33.5 & 24.4 & 33.6 \\
\hline il & 22.8 & 33.6 & 22.1 & 1.5 & 21.7 & 33.8 & 22.0 & 33.8 & 21.4 & 33.7 & 21.7 & 33.8 & 23.0 & 33.8 & 23.9 & 33.8 & 25.1 & \begin{tabular}{|l}
33.8 \\
\end{tabular} & \begin{tabular}{|l}
6.1 \\
\end{tabular} & \begin{tabular}{|l}
33.7 \\
\end{tabular} & \begin{tabular}{|l|l}
26.2 \\
\end{tabular} & 33.6 & 24.4 & 33.6 \\
\hline 15 & 212.8 & 33.6 & 22.0 & 33.6 & 21.5 & 33.8 & 21.8 & 33.8 & 22.1 & 33.8 & 21.3 & 33.8 & 21.5 & 33.9 & 23.2 & 33.9 & 24.3 & \begin{tabular}{|l}
33.9 \\
\end{tabular} & \begin{tabular}{|l}
15.4 \\
\end{tabular} & \begin{tabular}{|l}
33.8 \\
\end{tabular} & 26.0 & 33.7 & 24.4 & 33.6 \\
\hline 41 & 212.8 & 33.6 & 21.9 & 33.6 & 21.4 & 33.8 & 21.6 & 33.8 & 21.8 & 33.8 & 22.1 & 33.9 & 21.2 & 34.0 & 22.6 & 34.1 & 23.6 & \begin{tabular}{|l}
34.1 \\
\end{tabular} & \begin{tabular}{|l}
4.8 \\
\end{tabular} & \begin{tabular}{|l}
34.0 \\
\end{tabular} & \begin{tabular}{|l|l}
15.7 \\
\end{tabular} & 33.8 & 24.4 & 33.6 \\
\hline 45 & 22.8 & 33.6 & 21.9 & 33.6 & 21.3 & 33.9 & 21.4 & 33.9 & 21.6 & 33.9 & 21.9 & 34.0 & 21.9 & 34.2 & 22.1 & 34.2 & 23.0 & 34.2 & \begin{tabular}{|l}
24.2 \\
\end{tabular} & 34,1 & 25.4 & 33.9 & 24.4 & 33.6 \\
\hline il & 21.7 & 33.6 & 21.8 & 33.6 & 21.2 & 33.9 & 21.2 & 33.9 & 21.5 & 33.8 & 21.7 & 34.0 & 21.7 & 34.2 & 211.6 & 34.3 & 21.5 & \begin{tabular}{|l}
34.3 \\
\end{tabular} & \begin{tabular}{|l}
23.8 \\
\end{tabular} & \begin{tabular}{|l|l}
34.2 \\
\end{tabular} & [5.2. & 33.9 & 24.3 & $33 . ?$ \\
\hline 19 & 21.7 & 33.7 & 21.8 & 33.6 & \begin{tabular}{|l}
21.1 \\
\end{tabular} & 33.9 & 21.1 & \begin{tabular}{|l|l}
33.9 \\
\end{tabular} & 21.4 & 33.8 & 21.6 & 34.0 & 21.5 & 34.3 & 21.5 & 34.3 & 21.3 & \begin{tabular}{|l}
34.3 \\
\end{tabular} & 33.6 & \begin{tabular}{|l}
34.2 \\
\end{tabular} & (5.0.0 & 34.0 & 24.3 & 33.7 \\
\hline 61 & 21.7 & 33.7 & 21.7 & 33.6 & 21.0 & 33.9 & 11.0 & \begin{tabular}{|l}
33.8 \\
\end{tabular} & 21.3 & 33.8 & 21.5 & 34.0 & 21.5 & 34.3 & 21.3 & 34.3 & 22.1 & 34.4 & 13,4 & \begin{tabular}{|l}
34.3 \\
\end{tabular} & 24.9 & 34.0 & 24.3 & 33.7 \\
\hline 65 & 21.7 & 33.7 & 21.7 & 33.6 & 21.0 & 33.9 & 20.9 & 33.8 & 21.2 & 33.8 & 21.4 & 34.0 & 21.4 & 34.3 & 21.2 & 34.3 & 22.0 & 34.4 & \begin{tabular}{|l}
13.2 \\
\end{tabular} & \begin{tabular}{|l}
34.3 \\
\end{tabular} & 24.8 & 34.1 & 24.3 & 33.8 \\
\hline 71 & 12.1 & 33.7 & 21.7 & 33.9 & 20.9 & 33.9 & 20.9 & 33.8 & 21.2 & 33.8 & 21.4 & 34.1 & 21.4 & 34.3 & 21.1 & 34.3 & $\mid 21.8$ & 34.4 & \begin{tabular}{|l|l}
23.1 \\
\end{tabular} & 34.4 & 24.7 & 34.1 & 24.2 & 33.8 \\
\hline
\end{tabular}

Bảng 4: Xác định vận tốc âm $V$ theo 12 tháng (năm2006) tại vị trí $A$

\begin{tabular}{|c|c|c|c|c|c|c|c|c|c|c|c|c|}
\hline Tháng & 1 & 2 & 3 & 4 & 5 & 6 & 7 & 8 & 9 & 10 & 11 & 12 \\
\hline 0 & 1518.7 & 1515.3 & 1522.8 & 1527.2 & 1534.0 & 1540.2 & 1540.7 & 1540.4 & 1537.4 & 1536.2 & 1533.7 & 1525.9 \\
\hline 2 & 1518.7 & 1515.2 & 1522.0 & 1526.2 & 1533.2 & 1540.2 & 1540.7 & 1540.4 & 1537.5 & 1536.2 & 1533.8 & 1525.9 \\
\hline 4 & 1518.7 & 1515.0 & 1521.0 & 1525.3 & 1532.3 & 1540.3 & 1540.6 & 1540.5 & 1537.5 & 1536.3 & 1533.8 & 1525.9 \\
\hline 6 & 1518.7 & 1514.8 & 1520.3 & 1524.3 & 1531.5 & 1540.3 & 1540.7 & 1540.6 & 1537.5 & 1536.3 & 1533.9 & 1525.9 \\
\hline 8 & 1518.6 & 1514.6 & 1519.5 & 1523.3 & 1530.6 & 1540.3 & 1540.7 & 1540.7 & 1537.5 & 1536.4 & 1534.0 & 1525.9 \\
\hline 10 & 1518.6 & 1514.4 & 1518.7 & 1522.3 & 1529.7 & 1540.4 & 1540.8 & 1540.8 & 1537.5 & 1536.4 & 1534.0 & 1525.9 \\
\hline 15 & 1518.6 & 1514.1 & 1518.0 & 1521.5 & 1528.4 & 1540.0 & 1540.4 & 1540.8 & 1537.6 & 1536.6 & 1534.2 & 1526.0 \\
\hline 20 & 1518.7 & 1513.8 & 1517.7 & 1521.0 & 1527.3 & 1539.2 & 1539.7 & 1540.6 & 1537.5 & 1536.7 & 1534.4 & 1526.2 \\
\hline 25 & 1518.7 & 1513.8 & 1517.4 & 1520.5 & 1526.4 & 1538.6 & 1539.0 & 1540.3 & 1537.4 & 1536.7 & 1534.5 & 1526.3 \\
\hline 30 & 1518.8 & 1513.6 & 1517.0 & 1520.0 & 1525.8 & 1538.0 & 1538.5 & 1539.8 & 1537.3 & 1536.7 & 1534.4 & 1526.4 \\
\hline 35 & 1518.9 & 1513.8 & 1517.0 & 1519.8 & 1525.5 & 1537.3 & 1537.9 & 1539.1 & 1536.9 & 1536.3 & 1534.1 & 1526.4 \\
\hline 40 & 1518.9 & 1513.8 & 1516.8 & 1519.5 & 1525.0 & 1536.6 & 1537.1 & 1538.3 & 1536.4 & 1536.9 & 1533.7 & 1526.5 \\
\hline Vtb & 1518.7 & 1514.3 & 1519.0 & 1522.6 & 1529.1 & 1539.3 & 1539.7 & 1540.2 & 1537.3 & 1536.4 & 1534.0 & 1526.1 \\
\hline
\end{tabular}

âm tại bề mặt và mặt đáy thay đổi không nhiều, khoảng 2-3 m/s do vị trí $A$ nằm tại khu vực phía bắc Vịnh Bắc Bộ (độ sâu đáy biển khoảng $40 \mathrm{~m}$ ), nên nhiệt độ bề mặt và nhiệt độ mặt đáy thay đổi không nhiều so với các vị trí khác. (Xem bảng 5)
Giá trị vận tốc âm tại vị trí $B$ nhỏ nhất vào tháng $2\left(\mathrm{~V}_{\min }=1517.7 \mathrm{~m} / \mathrm{s}\right)$ và lớn nhất vào tháng 7 , tháng $8\left(V_{\max }=1541.2 \mathrm{~m} / \mathrm{s}\right)$ và biển đổi vận tốc âm trong năm tại vị trí $B$ (giữa Vịnh Bắc Bộ) là $23.5 \mathrm{~m} / \mathrm{s}$. Nếu so sánh vận tốc âm trung bình thì giá trị nhỏ 
Bảng 5: Xác định vận tốc âm V theo 12 tháng (năm 2006) tại vị trí $B$

\begin{tabular}{|c|c|c|c|c|c|c|c|c|c|c|c|c|}
\hline Tháng & 1 & 2 & 3 & 4 & 5 & 6 & 7 & 8 & 9 & 10 & 11 & 12 \\
\hline 0 & 1520.8 & 1518.5 & 1520.0 & 1529.4 & 1536.7 & 1539.7 & 1541.1 & 1514.2 & 1539.1 & 1536.7 & 1532.4 & 1527.4 \\
\hline 2 & 1520.8 & 1518.4 & 1519.9 & 1529.2 & 1536.7 & 1539.6 & 1541.0 & 1514.1 & 1539.2 & 1536.7 & 1532.5 & 1527.5 \\
\hline 4 & 1520.8 & 1518.3 & 1519.8 & 1529.0 & 1536.6 & 1539.5 & 1541.0 & 1514.1 & 1539.3 & 1536.7 & 1532.7 & 1527.6 \\
\hline 6 & 1520.8 & 1518.3 & 1519.7 & 1528.8 & 1536.4 & 1539.4 & 1540.9 & 1514.1 & 1539.4 & 1536.8 & 1532.9 & 1527.6 \\
\hline 8 & 1520.9 & 1518.2 & 1519.5 & 1528.5 & 1536.3 & 1539.3 & 1540.9 & 1514.1 & 1539.4 & 1536.8 & 1533.0 & 1527.7 \\
\hline 10 & 1520.9 & 1518.2 & 1519.4 & 1528.3 & 1536.1 & 1539.3 & 1540.9 & 1514.0 & 1539.5 & 1536.8 & 1533.0 & 1527.8 \\
\hline 15 & 1521.0 & 1518.0 & 1519.3 & 1527.4 & 1534.6 & 1537.6 & 1539.2 & 1539.9 & 1538.9 & 1536.8 & 1533.2 & 1528.0 \\
\hline 20 & 1521.0 & 1518.0 & 1519.2 & 1526.4 & 1532.9 & 1535.7 & 1537.3 & 1538.6 & 1538.4 & 1536.7 & 1533.3 & 1528.1 \\
\hline 25 & 1521.1 & 1517.9 & 1519.1 & 1525.2 & 1531.3 & 1534.0 & 1535.9 & 1537.5 & 1537.8 & 1536.5 & 1533.3 & 1528.2 \\
\hline 30 & 1521.1 & 1517.8 & 1528.8 & 1524.0 & 1529.9 & 1532.5 & 1534.6 & 1536.4 & 1537.1 & 1536.2 & 1533.4 & 1528.3 \\
\hline 35 & 1521.2 & 1517.9 & 1528.6 & 1523.5 & 1529.4 & 1532.0 & 1534.1 & 1535.6 & 1536.4 & 1535.9 & 1533.4 & 1528.3 \\
\hline 40 & 1521.2 & 1517.9 & 1528.5 & 1523.2 & 1529.0 & 1531.6 & 1533.9 & 1535.0 & 1535.7 & 1535.4 & 1533.5 & 1528.4 \\
\hline 45 & 1521.2 & 1517.9 & 1528.2 & 1522.8 & 1528.6 & 1531.3 & 1533.5 & 1534.5 & 1534.9 & 1534.8 & 1533.2 & 1528.4 \\
\hline 50 & 1521.2 & 1517.7 & 1527.9 & 1522.2 & 1528.2 & 1531.0 & 1533.2 & 1534.0 & 1534.3 & 1534.4 & 1533.1 & 1528.4 \\
\hline Vtb & 1521.0 & 1518.1 & 1529.1 & 1526.3 & 1533.0 & 1535.9 & 1537.7 & 1538.4 & 1537.8 & 1536.2 & 1533.1 & 1528.0 \\
\hline
\end{tabular}

nhất là tháng $2\left(\mathrm{~V}_{\mathrm{tb}}=1518.1 \mathrm{~m} / \mathrm{s}\right)$, lớn nhất chúng ta thấy:

là tháng $8(\mathrm{Vtb}=1538.4 \mathrm{~m} / \mathrm{s})$, chênh lệch vận tốc trung bình giữa tháng 2 và tháng 8 là $20,3 \mathrm{~m} / \mathrm{s}$.

Từ giá trị vận tốc âm trung bình chúng tôi vẽ biểu đồ thay đổi vận tốc âm trung bình theo 12 tháng tại vị trí $B$ (hình 5).

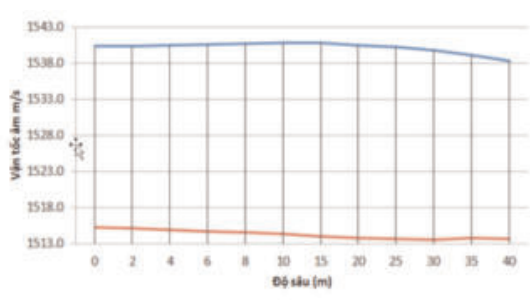

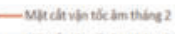

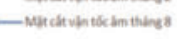

Hình 4: Mặt cắt vận tốc âm tháng 2 và tháng 8 (vị trí $B$ )

Theo mặt cắt tại thời điểm tháng 2 và tháng 8 (có độ chênh vận tốc âm lớn nhất)
Biên độ biến đổi vận tốc âm của tháng 8 khoảng $7 \mathrm{~m} / \mathrm{s}$ với thời điểm nhiệt độ cao nhất và có sự ảnh hưởng rõ rệt của nhiệt độ giữa lớp bề mặt và lớp đáy.

Biên độ vận tốc âm tại thời điểm tháng 2 biến đổi không nhiều do nhiệt độ tại vị trí quan trắc biến đổi không nhiều.

Từ số liệu mặt cắt vận tốc âm theo 2 tháng đặc trưng tại vị trí $B$ cho thấy ảnh hưởng của nhiệt độ tại khu vực này là đáng kể, nhiệt độ tại khu vực quan trắc biến đổi thất thường, khó xác định. (Xem bảng 6)

Giá trị vận tốc âm nhỏ nhất vào tháng 3 $\left(V_{\min }=1523.9 \mathrm{~m} / \mathrm{s}\right)$, lớn nhất vào tháng 7 , tháng $8\left(\mathrm{~V}_{\text {max }}=1541.2 \mathrm{~m} / \mathrm{s}\right)$ và biển đổi vận tốc âm trong năm tại vị trí C (Nam Vịnh Bắc 
Bảng 6: Xác định vận tốc âm V theo 12 tháng (năm 2006) tại vị trí C

\begin{tabular}{|c|c|c|c|c|c|c|c|c|c|c|c|c|}
\hline Tháng & 1 & 2 & 3 & 4 & 5 & 6 & 7 & 8 & 9 & 10 & 11 & 12 \\
\hline 0 & 1527.5 & 1526.2 & 1526.9 & 1532.2 & 1537.2 & 1540.1 & 1541.2 & 1541.2 & 1539.7 & 1539.3 & 1535.1 & 1531.1 \\
\hline 2 & 1527.5 & 1526.2 & 1526.9 & 1532.0 & 1536.9 & 1539.7 & 1540.9 & 1541.0 & 1539.7 & 1539.2 & 1535.3 & 1531.2 \\
\hline 4 & 1527.5 & 1526.1 & 1526.8 & 1531.8 & 1536.6 & 1539.4 & 1540.6 & 1540.8 & 1539.7 & 1539.1 & 1535.5 & 1531.2 \\
\hline 6 & 1527.6 & 1526.1 & 1526.7 & 1531.7 & 1536.3 & 1539.1 & 1540.3 & 1540.6 & 1539.7 & 1539.1 & 1535.8 & 1531.3 \\
\hline 8 & 1527.6 & 1526.1 & 1526.7 & 1531.5 & 1535.9 & 1538.7 & 1539.9 & 1540.3 & 1539.7 & 1539.0 & 1536.0 & 1531.3 \\
\hline 10 & 1527.6 & 1526.1 & 1526.6 & 1531.4 & 1535.6 & 1538.3 & 1539.5 & 1540.1 & 1539.7 & 1538.9 & 1536.2 & 1531.4 \\
\hline 15 & 1527.7 & 1526.0 & 1526.3 & 1530.0 & 1533.2 & 1535.0 & 1536.2 & 1537.7 & 1538.5 & 1538.5 & 1536.5 & 1531.6 \\
\hline 20 & 1527.8 & 1526.0 & 1526.1 & 1528.6 & 1530.8 & 1531.8 & 1533.0 & 1535.2 & 1537.1 & 1537.9 & 1536.5 & 1531.7 \\
\hline 25 & 1527.9 & 1525.9 & 1525.8 & 1527.3 & 1528.7 & 1529.5 & 1530.5 & 1532.9 & 1535.5 & 1537.0 & 1536.4 & 1531.9 \\
\hline 30 & 1527.9 & 1525.8 & 1525.2 & 1526.0 & 1527.0 & 1527.7 & 1528.5 & 1530.8 & 1533.6 & 1535.9 & 1536.3 & 1532.0 \\
\hline 35 & 1528.0 & 1525.8 & 1524.9 & 1525.4 & 1526.2 & 1527.0 & 1527.5 & 1529.2 & 1532.1 & 1534.7 & 1535.9 & 1532.0 \\
\hline 40 & 1528.0 & 1525.7 & 1524.6 & 1525.0 & 1525.8 & 1526.5 & 1526.8 & 1527.9 & 1530.6 & 1533.4 & 1535.3 & 1532.0 \\
\hline 45 & 1528.0 & 1525.7 & 1524.4 & 1524.7 & 1525.4 & 1526.1 & 1526.3 & 1526.8 & 1529.3 & 1532.2 & 1534.8 & 1532.0 \\
\hline 50 & 1528.1 & 1525.6 & 1524.2 & 1524.3 & 1525.0 & 1525.8 & 1525.9 & 1525.9 & 1528.2 & 1531.3 & 1534.4 & 1532.1 \\
\hline 55 & 1528.1 & 1525.6 & 1524.1 & 1524.1 & 1524.8 & 1525.6 & 1525.7 & 1525.6 & 1527.8 & 1530.9 & 1534.2 & 1532.1 \\
\hline 60 & 1528.2 & 1525.6 & 1524.0 & 1523.8 & 1524.6 & 1525.4 & 1525.6 & 1525.3 & 1527.4 & 1530.5 & 1534.0 & 1532.2 \\
\hline 65 & 1528.2 & 1525.6 & 1524.0 & 1523.7 & 1524.5 & 1525.3 & 1525.6 & 1525.1 & 1527.2 & 1530.3 & 1533.9 & 1532.3 \\
\hline 70 & 1528.3 & 1525.6 & 1523.9 & 1523.6 & 1524.4 & 1525.3 & 1525.6 & 1524.9 & 1526.9 & 1530.0 & 1533.8 & 1532.3 \\
\hline Vtb & 1527.9 & 1525.9 & 1525.5 & 1527.6 & 1529.9 & 1531.5 & 1532.2 & 1532.8 & 1534.0 & 1535.4 & 1535.3 & 1531.8 \\
\hline
\end{tabular}

Bộ) là $17.3 \mathrm{~m} / \mathrm{s}$. Nếu so sánh vận tốc âm khoảng $3 \mathrm{~m} / \mathrm{s}$. Với tháng có nhiệt độ cao trung bình thì giá trị nhỏ nhất vào tháng 3 , nhất (tháng 8), biên độ biến đổi vận tốc âm $\left(\mathrm{V}_{\mathrm{tb}}=1525.5 \mathrm{~m} / \mathrm{s}\right)$ và lớn nhất vào tháng 10 $\left(V_{\mathrm{tb}}=1535.4 \mathrm{~m} / \mathrm{s}\right)$, chênh lệch vận tốc âm trung bình giữa tháng 3 và tháng 8 là 9.9 $\mathrm{m} / \mathrm{s}$.

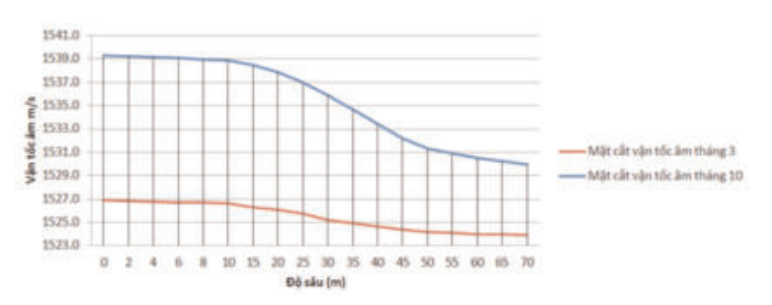

Hình 5: Mặt cắt vận tốc âm tháng 3 và tháng 8 (vị trí C)

Từ mặt cắt vận tốc âm tháng 3 và tháng 10 tại vị trí $\mathrm{C}$ ta thấy, biên độ biến đổi vận tốc âm tại tháng có nhiệt độ thấp nhất (tháng 3) khoảng $15 \mathrm{~m} / \mathrm{s}$. Tại vị trí này nhiệt độ bề mặt và mặt đáy biến đổi lớn nhưng khá tuyến tính. Từ $0 \mathrm{~m}$ đến $10 \mathrm{~m}$ độ sâu nhiệt độ bề mặt thay đổi không lớn. Từ độ sâu lớn hơn $10 \mathrm{~m}$, nhiệt độ bắt đầu biến đổi nhanh và đến lớp đáy nhiệt độ dần ổn định. Khi đó biến đổi của vận tốc âm chủ yếu phụ thuộc vào độ sâu.

\section{Phân tích kết quả tính toán}

Từ số liệu quan trắc và kết quả tính toán vận tốc âm, chúng tôi phân tích sự thay đổi của vận tốc âm ở khu vực biển Vịnh Bắc bộ theo một số cách tiếp cận như sau:

4.1. Ảnh hưởng của nhiệt độ 
Từ các bảng 1, 2, 3, 4, 5, 6 ta nhận thấy, nhiệt độ tại các lớp nước mặt $(z=0)$ thay đổi nhanh và lớn nhất so với các lớp nước khác và biến thiên vận tốc âm do nhiệt độ tại lớp nước $z=0$ khoảng $25 \mathrm{~m} / \mathrm{s}$. Như vậy các thiết bị thủy âm có sử dụng công nghệ đa chùm tia cần phải quan tâm tới vấn đề thay đổi tại lớp nước $z=0$. Khi tia sóng âm đi qua các lớp độ sâu, hiện tượng khúc xạ và tán xạ cùng với góc lan truyền giữa các lớp nước khác nhau với vận tốc âm khác nhau. Do đó, vận tốc âm bề mặt ảnh hưởng nhiều tới kết quả khảo sát thủy âm bằng thiết bị đa chùm tia

\section{2. Ảnh hưởng của độ mặn}

Theo các bảng thống kê tại các vị trí, các thời gian khác nhau độ mặn hầu như không biến thiên nhiều, chỉ nằm trong khoảng 0.6 ppt. Như vậy, độ mặn tại khu vực Vịnh Bắc bộ khá ổn định và ảnh hưởng tới vận tốc âm tại các lớp nước là rất nhỏ. Chỉ cần chú ý tới độ mặn bề mặt trong các trường hợp thời tiết thay đổi đột ngột như mưa lớn, bão hoặc các trường hợp làm thay đổi độ mặn tại lớp nước $\mathrm{z}=0$.

4.3. Sự biến đổi vận tốc âm theo mùa (theo thờ gian)

Sự biến đổi vận tốc âm theo mùa trong khu vực Vịnh Bắc bộ thể hiện rõ rệt khi thời tiết thay đổi từ mùa nóng sang mùa lạnh. Ảnh hưởng gây nên chủ yếu là nhiệt độ, sau đó đến độ sâu và cuối cùng là độ mặn. Sự biến thiên vận tốc âm theo mùa trong các lớp nước khoảng $27.3 \mathrm{~m} / \mathrm{s}$ phụ thuộc vào độ sâu và vị trí quan trắc.

4.4. Sự biến đổi vận tốc âm theo khu vực
Biến thiên theo vị trí quan trắc từ vĩ độ cao tới vĩ độ thấp tại các lớp nước $z=0$ khoảng $20-25 \mathrm{~m} / \mathrm{s}$, nhưng tại lớp nước đáy thay đổi khá lớn khoảng 7-25 m/s. Sự thay đổi này do ảnh hưởng một phần bởi nhiệt độ và độ sâu tại vị trí quan trắc. Ở khu vực phía Bắc Vịnh Bắc bộ, sự biến thiên vận tốc âm lớn xảy ra tại lớp nước mặt và lớp đáy, sau đó giảm dần về phía Nam. Do đó khi lựa chọn vị trí lấy mẫu vận tốc âm phục vụ khảo sát, cần xác định được độ sâu lớn nhất của khu đo và sự thay đổi địa hình của khu vực để có phương án bổ sung SSP cho phù hợp.

\section{Nhận xét và kết luận}

- Theo kết quả quan trắc tại các vị trí thuộc Vịnh Bắc bộ, vận tốc âm biến thiên theo hướng Bắc Nam. Phía Bắc biến thiên vận tốc âm nhiều hơn phía Nam và biên độ thay đổi của vận tốc âm tại phía Bắc cũng lớn hơn so với phía Nam. Sự biến đổi của nhiệt độ tại khu vực này ảnh hưởng nhiều tới vận tốc âm so với độ sâu và độ mặn. Đây là điều cần lưu ý để khai thác có hiệu quả các thiết bị đo đạc sử dụng nguyên lý thủy âm.

- Sự thay đổi nhiệt độ nước biển giúp kiểm soát mặt cắt vận tốc âm SSP trong nước biển và đại dương do ảnh hưởng của độ mặn tới vận tốc âm là không đáng kể. Đây là điều cần lưu ý để khai thác có hiệu quả các thiết bị đo đạc sử dụng nguyên lý thủy âm.

- Tại các vị trí khác nhau, độ sâu khác nhau sự biến thiên vận tốc âm cũng khác nhau, nên với khu đo có diện tích rộng hoặc trải dài cần phải có phương án xác định bổ sung thêm SSP. Đặc biệt, tại các vị trí có sự 
thay đổi lớn về nhiệt độ, độ sâu, độ mặn như khu vực cửa sông, khu vực độ sâu đột biến cần thiết phải xác định bổ sung SSP, vì lúc này giá trị các tham số xác định vận tốc âm đã bị biến đổi.

- Với các thiết bị thủy âm sử dụng nguyên lý đa chùm tia, nếu thời tiết thay đổi hoặc độ sâu của khu vực khảo sát biến đổi lớn cũng cần xác định bổ sung SSP do sự biến thiên vận tốc âm trong cột nước lúc này là đáng kể.O

\section{Tài liệu tham khảo}

[1]. Phạm Văn Thục. Âm học biển và trường sóng âm tại khu vực Biển Đông Việt Nam. Nhà xuất bản Khoa học tự nhiên và công nghệ, Hà Nội 2011.

[2]. Xavier Lurton. An Introduction to underwater acoustics - principles and applications. Springer-2002.

[3]. K. V. Mackenzie, "Nine-term equation for the sound speed in the oceans," J. Acoust. Soc. Am., vol. 70, no. 3, pp. 807812, 1981.

pp. $712,1998.0$
[4]. A.B. Coppens, "Simple equations for the speed of sound in Neptunian waters," J. Acoust. Soc. Am., vol. 69 , no. 3, pp. 862863, 1981.

[5]. V.A. Del Grosso, "New equation for the speed of sound in natural waters (with comparisons to other equations)," J. Acoust. Soc. Am., vol. 56, no. 4, pp. 1084-1091, 1974.

[6]. C. C. Leroy and F. Parthiot, "Depthpressure relationships in the oceans and seas," J. Acoust. Soc. Am. vol. 103, pp. 1346-1352, 1998.

[7]. W. Wilson, "Equation for the speed of sound in Sea Water," J. Acous. Soc. Am., vol. 32, no. 10, pp.1357, 1990.

[8]. C.T. Chen, and F. J. Millero, "Speed of sound in seawater at high pressures," J. Acoust. Soc. Am., vol. 62, no. 5, pp.11291135, 1977.

[9]. H. Medwin, and C. S. Clay, "Fundamentals of Acoustical Oceanography," Academic Press: London,

\section{Summary}

\section{Influence of temperature on sound speed in the Gulf of Tonkin}

Nguyen Van Cuong, Center for Sea Survey and Mapping

Nguyen Gia Trong, University of Mining and Geology

Sound speed in the oceans depends on temperature, salinity, and pressure and has large seasonal and spatial variations. The article presents results of negative velocity surveys at some locations in Tonkin Gulf with data on temperature and salinity at depths from $0 \mathrm{~m}$ to $70 \mathrm{~m}$, from $01 / 01 / 2006$ to $01 / 12 / 2006$. The results show that variation in velocity during one year in Tonkin Gulf was $27.2 \mathrm{~m} / \mathrm{s}$. This is important in determining the velocity of sound when using underwater sound wave technology in the Tonkin Gulf. $O$ 